Revista de Investigación de Física 21(1), 182101752 (2018)

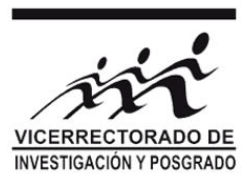

\title{
¿Física Teórica y Octoniones?
}

\author{
Edgar Vera ${ }^{*}$ \\ ${ }^{1}$ Universidad Nacional Mayor de San Marcos, Lima - Perú
}

Recibido 02 febrero 2018 - Aceptado 30 abril 2018

\begin{abstract}
Presentamos un tema, de interés común para Docentes Universitarios de Física y Matemática, que permite reactualizar el aspecto unificado y unificador de la Matemática y su estrecha relación con la Física. El objetivo final es tornarlo accesible a los estudiantes de pregrado.

Palabras claves: Cuaterniones, Octoniones, Álgebras Reales (los escalares de la estructura vectorial son los reales), Álgebra Geométrica.
\end{abstract}

\section{Octonions and Theoretical Physics?}

We present a topic of common interest to university professors of physics and mathematics, which allows to revisit the unified aspects of mathematics and its close relationship whith physics.

Keywords: Quaternions, Octonions, Real Algebra (the scalars of the vector structure are the reals), Geometric Algebra.

\section{Introducción}

En este siglo XXI ha cobrado fuerza la conjetura de que los Octoniones serían de utilidad en la Física Teórica. John Baez y John Huerta los relacionan con la Teoría de Cuerdas y Supersimetría en The Strangest Numbers in String Theory [2].

\section{Fundamento Teórico}

Octoniones, presentados por Graves en 1843 y por Cayley en 1844 , es el nombre del miembro más joven de una familia de cuatro álgebras reales:

Reales, Complejos, Cuaterniones y Octoniones.

Se trata de una familia de álgebras donde es posible medir y dividir (llamadas por ello Álgebras de División Normadas). En 1898 Hurwitz demostró que estas son las únicas álgebras de división normadas y tienen $\mathbb{R}$-dimensiones $1,2,4$ y 8 , respectivamente (ver [1] ).

Las álgebras de los reales $\mathbb{R}$ y los complejos $\mathbb{C}$ eran usadas, sin una definición precisa, en el siglo XVIII. En 1835, al percatarse que los complejos permiten algebrizar el proceso geométrico de rotación en $\mathbb{R}^{2}$, Hamilton busca denodadamente un álgebra que haga lo propio en $\mathbb{R}^{3} \mathrm{y}$, en octubre de 1843 , crea el álgebra no conmutativa de los cuaterniones $\mathbb{H}$. En diciembre de ese mismo año su amigo
Graves le remite un escrito sobre el álgebra no conmutativa y no asociativa de los Octoniones $\mathbb{O}($ ver [1] ).

\section{Antecedentes}

En 1679, después de conocer la Geometría Analítica creada por Descartes y Fermat, Leibnitz le manifiesta a Huygens (ver 3] ):

\section{la Física no podrá avanzar más a no ser que se encuentre un nuevo método de análisis más geométrico, que permita expresar y operar con direcciones tan directamente como el álgebra (de los números reales) representa y opera con las magnitudes (el concepto de longitud en la geometría analítica). (traducción del autor)}

Leibnitz, en la búsqueda de un modelo matemático apropiado para el desarrollo de la Física, entreveía el Álgebra Geométrica creada por Clifford entre los años 1873 y 1879 conjugando las álgebras de Hamilton (1843) y de Grassmann (1844). El Álgebra Geométrica unifica y simplifica el estudio y aplicación de los Complejos y Cuaterniones (ver [6] y [7]).

En el siglo XX, en 1920, Heisenberg manifiesta que la Física requiere una matemática completamente nueva que incluya álgebras no conmutativas. En respuesta a esto Pauli y Dirac recrean el Algebra Geométrica recurriendo a

\footnotetext{
*edverasar@gmail.com
} 
Álgebras de Matrices. En 1966 aparece el aporte de Hestenes [4] y [5]. (un "álgebra de matrices sin matrices").

\section{Metodología}

Presentamos los Cuaterniones y Octoniones, estos últimos también conocidos como números de Cayley, replicando el proceso tradicional usado para introducir los Complejos (ver [1] y [6]):

1. Como sabemos, considerando la conocida raíz cuadrada de -1 , $i$, y pares de reales (los Espinores $\mathrm{Bi}$ dimensionales de Pauli) se presenta:

$$
\mathbb{C}=\{r+s i ; \quad r, s \in \mathbb{R}\} .
$$

2. Considerando ahora otra raíz cuadrada de $-1, j$, y pares de complejos (Ios Espinores de Pauli) presentamos:

$$
\mathbb{H}=\{a+b j ; \quad a, b \in \mathbb{C}\}
$$

3. Finalmente los Octoniones o números de Cayley se presentan considerando una nueva raíz cuadrada de $-1, l$, y pares de cuaterniones (relacionados con los Espinores de Dirac)

$$
\mathbb{O}=\{A+B l ; \quad A, B \in \mathbb{H}\} .
$$

\begin{tabular}{c|c|c|c|c|c|c|c} 
& $i$ & $j$ & $k$ & $l$ & $m$ & $n$ & $o$ \\
\hline$i$ & -1 & $k$ & $-j$ & $m$ & $-l$ & $-o$ & $n$ \\
\hline$j$ & $-k$ & -1 & $i$ & $n$ & $o$ & $-l$ & $-m$ \\
\hline$k$ & $j$ & $-i$ & -1 & $o$ & $-n$ & $m$ & $-l$ \\
\hline$l$ & $-m$ & $-n$ & $-o$ & -1 & $i$ & $j$ & $k$ \\
\hline$m$ & $l$ & $-o$ & $n$ & $-i$ & -1 & $-k$ & $j$ \\
\hline$n$ & $o$ & $l$ & $-m$ & $-j$ & $k$ & -1 & $-i$ \\
\hline$o$ & $-n$ & $m$ & $l$ & $-k$ & $-j$ & $i$ & -1
\end{tabular}

Tabla 1: Tabla del producto de $\mathbb{C}, \mathbb{H}$ y $\mathbb{O}$

\section{Resultados y Discusión}

Veamos que el álgebra de los Complejos y las álgebras hipercomplejas de los Cuaterniones y Octoniones son extensiones del álgebra de los Reales y determinan el siguiente encaje de álgebras reales:

$$
\mathbb{R}<\mathbb{C}<\mathbb{H}<\mathbb{O} \text {. }
$$

Usaremos para ello la Tabla 1, una adaptación de la tabla dada en [1].

Esta Tabla, además de determinar el producto de $\mathbb{C}, \mathbb{H}$ y $\mathbb{O}$ establece lo siguiente:

1. $k=i j, m=i l, n=j l$, y $o=j m$, esto implica que la raices de $-1 i, j$, y $l$ determinan las otras cuatro.

2. $j i=-i j$, esto implica que el producto de cuaterniones y octoniones no es conmutativo.

3. $(i j) l=-i(j l)$, esto implica que el producto de octoniones no es asociativo.

Justifiquemos, usando la Tabla 1 , el encaje de álgebras (3) partiendo de que el álgebra conmutativa de los Complejos se expresa de la forma

$$
\mathbb{C}=\left\{r_{0}+r_{1} i ; \quad r_{j} \in \mathbb{R}\right\} .
$$

En efecto, reemplazando $a=r_{0}+r_{1} i$ y $b=r_{2}+r_{3} i$ en (1), distribuyendo y simplificando usando exclusivamente la Tabla 1, obtenemos:

$$
\mathbb{H}=\left\{r_{0}+r_{1} i+r_{2} j+r_{3} k ; \quad r_{j} \in \mathbb{R}\right\} .
$$

De modo similar, reemplazando $A=r_{0}+r_{1} i+r_{2} j+r_{3} k$ y $B=r_{4}+r_{5} i+r_{6} j+r_{7} k$ en (2), obtenemos

$\mathbb{O}=\left\{r_{0}+r_{1} i+r_{2} j+r_{3} k+r_{4} l+r_{5} m+r_{6} n+r_{7} O ; r_{j} \in \mathbb{R}\right\}$.

Continuando, (4) y (5) son claramente espacios vectoriales.

Finalmente, para obtener las estructuras de álgebra, los productos en (4) y (5) se procesan distribuyendo y simplificando con la única ayuda de la Tabla 1 (sin usar la conmutatividad ni la asociatividad de los polinomios) hasta obtener una expresión del mismo tipo. Este procedimiento para realizar los productos en $\mathbb{H}$ y $\mathbb{O}$ se sustenta en el Método de Cayley-Dickson que generaliza la idea que Hamilton utilizó, en 1835, para formalizar el álgebra de los complejos $\mathbb{C}$ como pares ordenados de números reales.

Es importante resaltar que, si bien las expresiones de los elementos de $\mathbb{H}$ y $\mathbb{O}$ en (4) y (5) corresponden a polinomios de primer grado (en las variables $i, j, k, l, m, n \mathrm{y}$ o) y la distributividad usada es similar al caso de polinomios, el producto que estamos considerando, mediante la 
tabla arriba, es una generalización del conocido producto de polinomios porque no es conmutativo ni asociativo en todos los casos.

\section{Conclusiones}

1. Ofrecemos una presentación unificada de las Álgebras de División Normadas: Dos conmutativas y asociativas (los Reales y los Complejos), una no conmutativa pero si asociativa (los Cuaterniones) y una no conmutativa ni asociativa (los Octoniones).

2. Proponemos la inclusión del Álgebra Geométrica en la formación básica de los Docentes Universitarios de Física y Matemática, una parte de la matemática iniciada en el siglo XIX y desarrollada en el siglo $X X$, sumamente importante por su relación con las Teorías de la Relatividad y la Física Cuántica.
3. Lo anterior tornaría factible participar en el reto que significan las Teorías de Unificación; en efecto, si bien el Algebra Geométrica es asociativa y el álgebra de los Octoniones no asociativa (es alternativa, como se puede ver en [1]) resulta viable obtener una generalización apropiada del concepto de Álgebra Geométrica para incluir a los Octoniones en el esquema conformado por los Complejos y Cuaterniones.

\section{Agradecimientos}

1. A los colegas de la Facultad de Ciencias Físicas que me acogieron en el Grupo de Física Teórica.

2. De igual forma a los colegas del Grupo de Ecuaciones Diferenciales, Análisis y Aplicaciones de la Facultad de Ciencias Matemáticas.

\section{Referencias}

[1] J. Baez. Octonions; Bulletin of the American Mathematical Society, vol.39, (2002).

[2] J. Baez y J. Huerta. The Strangest Numbers in String Theory; Scientific American, (2011).

[3] O. Bekken. Wessel on Vectors; A Workshop at the History of Mathematics, Norway, (1988).
[4] D. Hestenes. Space-Time Algebra; Gordon and Breach, (1966).

[5] D. Hestenes. New Foundations for Classical Mechanics; Kluwer Academic Publishers, (1993).

[6] C. Perwass. Geometric Algebra with Applications in Engineering; Springer, (2009).

[7] J. Snigg. Clifford Algebra, A Computacional tool for Physicists; Oxford University Press, (1997). 
